\title{
Invitro Haploid Plantlet Regeneration through Anther Culture in Locally Adapted Cultivar of Indian Mustard (Brassica juncea L. Czern and Coss)
}

\author{
N. Reetisana ${ }^{1}$, Th. Renuka Devi ${ }^{1 *}$, H. Nanita Devi' ${ }^{2}$ J.M. Laishram ${ }^{1}$ \\ and Artibashisha Hijam Pyngrope ${ }^{1}$ \\ ${ }^{1}$ College of Agriculture, Central Agricultural University, Iroisemba, Imphal, \\ Manipur (795 004), India \\ ${ }^{2}$ AICRP (Soybean), Directorate of Research, C. A. U., Lamphelpat, Imphal, \\ Manipur (795 004), India
}

*Corresponding author

\begin{abstract}
A B S T R A C T
Keywords

Anther culture,

Angrogenic,

Brassica juncea, in-

vitro regeneration

Article Info

Accepted:

04 March 2018

Available Online:

10 April 2018

Anthers from four genotype of Indian mustard, namely Local Yella (CAULC-1), JD-6, Kranti and NDRE-22) were cultured in vitro to observe their androgenic responses. Different concentrations and combinations of growth regulators were supplemented in $\mathrm{B}_{5}$ and MS medium. The range of callus induction was between 16.11 and $66.39 \%$. The maximum rate of callus induction in terms of percentage (\%) was observed in $\mathrm{MS}+3 \mathrm{ml} / \mathrm{l}$ 2, 4-D + 1ml/1 BAP (66.39). Among the Genotypes CAULC-1 (Local Yella) showed the best performance for days to callus initiation and per cent of callus induction while NDRE22 was the poorest. Maximum per cent of shoot initiation at 30 and 45 DAI $(55.55 \%$ and $59.44 \%$ respectively) was observed in $\mathrm{MS}+4 \mathrm{ml} / \mathrm{l} \mathrm{BAP}+1 \mathrm{ml} / \mathrm{l} \mathrm{NAA}$. Local Yella (CAULC-1) recorded the best for shoot regeneration followed by Kranti. The media composition $\mathrm{MS}+1 \mathrm{ml} / \mathrm{l} \mathrm{NAA}+0.5 \mathrm{ml} / 1 \mathrm{BAP}$ showed the minimum days (12.97) and highest per cent of root initiation $(45.83 \%)$ and also maximum length of regenerated root $(3.42 \mathrm{~cm})$ respectively. Local Yella was found to be the best genotype which took minimum time (12.44 days). Regeneration of haploid plants was confirmed by cytological examinations of the root tips of the plantlets from the callus of anther culture. The present study can be concluded that Local Yella (CAULC-1) showed better androgenic response than other genotypes under study.
\end{abstract}

\section{Introduction}

Rapeseed - mustard is one of the most important edible oil crops which accounts for $22.6 \%$ of the total oil produced and the production ranks second among all the oil crops in India (Anonymous, 2013). Oil of Indian mustard (Brassica juncea) is consumed in large quantity and it covers $85-90 \%$ of the total area under cultivation of rapeseedmustard in India (Anonymous, 2013). In Manipur, the crop is grown just after the harvest of rice but productivity is far below the national level resulting into low production. Among the locally grown genotypes, Local Yella (CAULC-1) is one of them which is without any genetic intervention. Against this, background 
attempts were made to provide several embryos and plantlets for locally adapted genotypes and early maturing mustard varieties. For a better breeding programme, it should have broad genetic base. The existing genetic base we have is not enough to meet the challenge.

To create variability and for their utilization, it is necessary to go for hybridization and selection of desirable types from the succeeding generation. Traditionally, homozygosity of the cross products is usually achieved by self-fertilization which is a time consuming process (Morrison and Evans, 1988).

To this regard, haploid production through anther culture along with double haploid (DH) production technique can play a significant role to reach the homozygosity in a shorter period of time. In vitro haploid production technique through anther culture provides rapid development of homozygous lines and has potential for more application as a versatile genetic manipulation tool for evaluation of desirable genotypes. Anther culture and subsequent plant regeneration offer an alternative and efficient technique to conventional breeding method and enable production of several plants from single anther.

It has also attracted considerable attention as a supplementary tool for the production of inbred lines and for obtaining hybrid cultivars. But this in vitro regeneration of Brassica through anther culture technique is used quite limitedly in our country.

So, there is indispensable need for studying the anther culture technique for improvement of Brassica. Therefore, the present investigation was undertaken to observe the androgenic responses of Indian mustard genotypes to anther culture.

\section{Materials and Methods}

\section{Materials}

Local genotype of Indian mustard, Local Yella (CAULC-1) along with JD-6, Kranti, and NDRE-22 were used for the present study and the seed materials collected from Department of Plant Breeding and Genetics, College of Agriculture, Central Agricultural University, Imphal, Manipur. Different media compositions were used to study the regeneration potentiality.

\section{Media used for anther culture}

$\mathrm{B}_{5}$ and MS media supplemented with 1.0, 2.0 and $3.0 \mathrm{ml} / \mathrm{l}$ 2,4-D along with same concentration of $1.0 \mathrm{ml} / \mathrm{l} \mathrm{BAP}$ were used for callus induction. Likewise, both the media $\mathrm{B}_{5}$ and MS supplemented with 4.0, 5.0, $6.0 \mathrm{ml} / 1$ BAP along with the constant concentration of $1.0 \mathrm{ml} / 1 \mathrm{NAA}$ were used for shoot initiation, while for root initiation, both the media supplemented with 1.0, 2.0 and $3.0 \mathrm{ml} / 1 \mathrm{NAA}$ with constant addition of $0.5 \mathrm{ml} / \mathrm{l} \mathrm{BAP}$ were used.

\section{Anther culture technique}

The unopened flower buds of all the four genotypes were collected when the microspores were at early to late uninucleate stage before 10 a.m. The collected flower buds were wrapped in the aluminium foil and kept in the refrigerator at $4{ }^{\circ} \mathrm{C}$ for 24 hours. After the cold treatment, they were surface sterilised under aseptic conditions in a laminar air flow chamber. They were rinsed in 0.1 per cent $\mathrm{HgCl}_{2}$ for 1 minute with intermittent shaking followed by three washings with sterile distilled water. The flower buds were opened with the help of sterile forceps and the six anthers were clipped off from each floret without damaging the anther wall and inoculated on sterile test tubes and incubated 
at $25^{\circ} \pm 1^{\circ} \mathrm{C}$ temperature in complete dark condition for callus induction and checked to record the response. Thirty anthers of each genotype were inoculated into each treatment. Six to seven weeks after inoculation of anthers, the regenerated calli attained convenient size. Then they were removed aseptically from the existing medium to a sterilised petriplate and cut into pieces and placed them into the test tubes with shoot initiation media and incubated in $25^{\circ} \mathrm{C} \pm 1^{\circ} \mathrm{C}$ with 16 hours photoperiod. Repeated subcultures were done at an interval of 15 days. When the sub-cultured calli were proliferated and differentiated into shoots and grew up to $2-3 \mathrm{~cm}$ in length, they were rescued aseptically and were separated from each other and again cultured on test tubes with freshly prepared root induction medium to induce root. Day to day observation was carried out to note the response of the growing plantlets.

\section{Cytological analysis}

The ploidy level of the plant regenerated from anther culture was investigated by standard acetocarmine root tip squashing technique (Darlington and Lacour, 1976). The $0.5 \mathrm{~cm}$ long root tips were fixed in 1:3 (glacial: ethanol) by volume for $24 \mathrm{hrs}$. The fixed root tips were washed thoroughly in running water to wash out the fixative. Then, the washed root tips were preserved in $70 \%$ ethanol for used. A root tip was taken in a clean slide and milky meristematic tissue was retained and all other parts were discarded and the tissue was allowed to stain for 20 minutes in $20 \%$ acetocarmine stain. The stained tissue was softened with $0.1 \mathrm{~N} \mathrm{HCl}$ for 50 seconds and warmed over spirit lamp and the tissue was squashed with a needle and a cover slip was placed over it. Then a filter paper was placed over the coverslip and gentle pressure was applied for further flattening and spreading of the chromosome and observed under Olympus phase contrast microscope at $100 \mathrm{X}$.

\section{Parameters recorded}

In callus induction, the parameters recorded were the days to callus induction, per cent of callus induction at 10, 20 and 30 days after inoculation (DAI). For shoot and root initiation, the parameters recorded were days to shoot and root initiation, per cent of shoot and root initiation at 15,30 and 45 DAI and length of regenerated shoot and root at 30,45 and 60 DAI respectively.

\section{Statistical analysis}

The data on various parameters of callus induction, shoot and root initiation were analysed in Factorial Randomised Block Design (FRBD) to obtain the effect of various treatments, genotypes and their interaction. Wherever necessary, data pertaining to different parameters were subjected to angular and square root transformation.

\section{Results and Discussion}

\section{Callus induction}

Callus induction performances of the genotypes, treatment compositions and genotype $\times$ interaction were evaluated and the results are presented in Tables 1,2 and 3 respectively. The two factors viz., genotypes and treatment compositions were found to be statistically significant for the parameter of days to callus induction and per cent of callus induction after 30 days of inoculation. Out of the four genotypes, days required for callus induction was minimum in Local Yella (25.61) followed by JD-6 (27.01). On the other hand, genotype NDRE-22 took more number of days for callus induction (28.48). The differential response of different genotypes for days to callus induction were also reported by Alam et al., (2009), Khan et al., (2009) and Sayem et al., (2010). In respect of the per cent of callus induction, Local Yella 
produced maximum calli $(53.67 \%)$ which was significantly higher than all other genotypes. It was followed by JD-6 and Kranti which gave callus induction of $51.59 \%$ and $49.08 \%$ respectively and the minimum was observed in NDRE-22 (37.96\%). Among the six treatment compositions of the media containing different concentrations of the phytohormones, $\mathrm{B}_{5}+3 \mathrm{ml} / \mathrm{l} 2,4-\mathrm{D}+1 \mathrm{ml} / \mathrm{l}$ BAP required minimum time (25.86 days) for callus induction which was found to be statistically at par with MS + $3 \mathrm{ml} / \mathrm{l} 2,4-\mathrm{D}+1$ $\mathrm{ml} / \mathrm{l}$ BAP (26.20 days). Both $\mathrm{MS}+1 \mathrm{ml} / \mathrm{l}$ BAP (28.00 days) and $\mathrm{B}_{5}+1 \mathrm{ml} / \mathrm{l} 2,4-\mathrm{D}+1$ $\mathrm{ml} / \mathrm{l}$ BAP (28.58 days) took maximum time for callus induction which were found to be statistically at par with each other. For the per cent of callus induction, MS + $3 \mathrm{ml} / \mathrm{l} 2,4-\mathrm{D}+$ $1 \mathrm{ml} / \mathrm{l} \mathrm{BAP}$ was the most responsive medium with an average of $66.39 \%$ callus induction followed by $\mathrm{B}_{5}+3 \mathrm{ml} / \mathrm{l}$ 2,4-D + $1 \mathrm{ml} / \mathrm{l} \mathrm{BAP}$ $(63.06 \%)$. As the concentration of $2,4-\mathrm{D}$ was decreased, there was a decrease in callus induction.

The minimum percentage of callus (16.11\%) was recorded in MS + $1 \mathrm{ml} / \mathrm{l} 2,4-\mathrm{D}+1 \mathrm{ml} / \mathrm{l}$ BAP. From the results, it was observed that the performance of media with these concentrations of 2,4-D were significantly different suggesting that both days to callus induction and per cent of callus induction were greatly influenced by the concentrations of 2,4-D used. The result is in consonance with the results of Narasimhulu and Chopra (1987), Ockenden and Mc Cleriaghan (1993) and Sayem et al., (2010). The genotype $\times$ treatment interaction was found to be significant for days to callus induction. Early callusing was found in the interaction of Local Yella with $\mathrm{B}_{5}+3 \mathrm{ml} / \mathrm{l}$ 2,4-D + $1 \mathrm{ml} / \mathrm{l} \mathrm{BAP}$ (23.33 days) which was found to be statistically at par with $\mathrm{MS}+3 \mathrm{ml} / \mathrm{l} 2,4-\mathrm{D}+1$ $\mathrm{ml} / \mathrm{l}$ BAP (23.78 days). Similar results were reported by Khan et al., (2009) and Sayem et al., (2010). But for per cent of callus induction, the interaction was found to be nonsignificant suggesting that all the four genotypes performed and responded equally in all the treatments under study which was found in agreement with the findings of Alam et al., (2009).

\section{Shoot initiation}

Significant variations were observed among the genotypes and treatments for days to shoot initiation. Among the genotypes, Local Yella started shoot initiation early (24.59 days) as compared to other genotypes JD-6 (26.63 days), Kranti (27.41 days) and NDRE-22 (28.85 days) as shown in Table 4. The results also indicated that the media containing $\mathrm{B}_{5}+4$ $\mathrm{ml} / \mathrm{l} \mathrm{BAP}+1 \mathrm{ml} / \mathrm{l}$ NAA required minimum time for shoot initiation (24.47 days). On the other hand, both $\mathrm{MS}+6 \mathrm{ml} / \mathrm{l} \mathrm{BAP}+1 \mathrm{ml} / \mathrm{l}$ NAA (28.70 days) and $\mathrm{B}_{5}+6 \mathrm{ml} / \mathrm{l} \mathrm{BAP}+1$ $\mathrm{ml} / \mathrm{l}$ NAA (28.36 days) took maximum time for shoot initiation (Table 5). Similar results were also revealed by Khan et al., (2009) and Sayem et al., (2010); who, found significant variations among the media with different hormonal concentrations. The genotype $\times$ treatment interaction was found to be significant for days to shoot initiation. Local Yella $\times \mathrm{B}_{5}+4 \mathrm{ml} / \mathrm{l} \mathrm{BAP}+1 \mathrm{ml} / \mathrm{l} \mathrm{NAA}$ took the lowest time (22.78 days), whereas, NDRE$22 \times \mathrm{B}_{5}+6 \mathrm{ml} / \mathrm{l} \mathrm{BAP}+1 \mathrm{ml} / \mathrm{l} \mathrm{NAA}$ took the highest time for shoot initiation (30.11 days) which is in concordance with the findings of Sayem et al., 2010 that revealed significant differences among the interaction (Table 6). The per cent of shoot initiation was significant at 30 and 45 DAI for genotypes, treatments and their interaction. For genotypes, the regeneration performances were found better in Local Yella $(51.48 \%)$ followed by Kranti $(48.15 \%)$ at 30 DAI. At 45 DAI, it was found better in Kranti (54.82\%) followed by Local Yella (54.07\%). Different concentrations of BAP showed significant variations for per cent of shoot initiation. 
Table.1 Response of genotypes on callus induction

\begin{tabular}{|c|c|c|c|c|}
\hline \multirow{2}{*}{ Genotypes } & $\begin{array}{c}\text { Days to callus } \\
\text { induction }\end{array}$ & 10 DAI & 20 DAI & Per cent (\%) callus induction after \\
\hline Local Yella & 25.61 & 0.00 & 0.00 & $53.67(47.73)$ \\
\hline Kranti & 27.96 & 0.00 & 0.00 & $49.08(44.20)$ \\
\hline JD-6 & 27.01 & 0.00 & 0.00 & $51.59(44.83)$ \\
\hline NDRE-22 & 28.48 & 0.00 & 0.00 & $37.96(37.57)$ \\
\hline S.Ed. $( \pm)$ & 0.25 & - & - & 1.06 \\
C.D. (0.05) & 0.50 & - & - & 2.14 \\
\hline
\end{tabular}

Figures in parenthesis are angular transformed values.

Table.2 Performance of media with different concentrations of phytohormones for callus induction

\begin{tabular}{|c|c|c|c|c|c|}
\hline \multirow[t]{2}{*}{ Treatment } & \multirow[t]{2}{*}{ Treatment composition } & \multirow{2}{*}{$\begin{array}{l}\text { Days to callus } \\
\text { induction }\end{array}$} & \multicolumn{3}{|c|}{ Per cent $(\%)$ of callus induction after } \\
\hline & & & 10 DAI & 20 DAI & 30 DAI \\
\hline $\mathbf{T}_{1}$ & $\mathrm{MS}+1 \mathrm{ml} / 12,4-\mathrm{D}+1 \mathrm{ml} / 1 \mathrm{BAP}$ & 28.00 & 0.00 & 0.00 & $16.11(23.53)$ \\
\hline$\overline{T_{2}}$ & $\mathrm{MS}+2 \mathrm{ml} / 12,4-\mathrm{D}+1 \mathrm{ml} / 1 \mathrm{BAP}$ & 27.58 & 0.00 & 0.00 & $60.56(51.70)$ \\
\hline $\mathbf{T}_{3}$ & $\mathrm{MS}+3 \mathrm{ml} / 12,4-\mathrm{D}+1 \mathrm{ml} / 1 \mathrm{BAP}$ & 26.20 & 0.00 & 0.00 & $66.39(54.66)$ \\
\hline $\mathbf{T}_{4}$ & $\mathrm{~B}_{5}+1 \mathrm{ml} / 1 \mathrm{2}, 4-\mathrm{D}+1 \mathrm{ml} / 1 \mathrm{BAP}$ & 28.58 & 0.00 & 0.00 & $25.10(28.83)$ \\
\hline $\mathbf{T}_{5}$ & $\mathrm{~B}_{5}+2 \mathrm{ml} / 12,4-\mathrm{D}+1 \mathrm{ml} / \mathrm{l} \mathrm{BAP}$ & 27.39 & 0.00 & 0.00 & $56.34(49.55)$ \\
\hline $\mathrm{T}_{6}$ & $\mathrm{~B}_{5}+3 \mathrm{ml} / 12,4-\mathrm{D}+1 \mathrm{ml} / 1 \mathrm{BAP}$ & 25.86 & 0.00 & 0.00 & $63.06(53.21)$ \\
\hline & $\begin{array}{c}\text { S.Ed. }( \pm) \\
\text { C.D. }(0.05)\end{array}$ & $\begin{array}{l}0.30 \\
0.61\end{array}$ & $\begin{array}{l}- \\
-\end{array}$ & $\begin{array}{l}- \\
-\end{array}$ & $\begin{array}{l}1.30 \\
2.62\end{array}$ \\
\hline
\end{tabular}

Table.3 Effect of genotype $\times$ treatment on callus induction

\begin{tabular}{|c|c|c|c|c|}
\hline Treatment & Treatment composition & Genotypes & $\begin{array}{l}\text { Days to callus } \\
\text { induction }\end{array}$ & $\begin{array}{l}\text { Per cent }(\%) \text { callus induction } \\
\text { after } 30 \text { DAI }\end{array}$ \\
\hline $\mathbf{T}_{1}$ & $\mathrm{MS}+1 \mathrm{ml} / \mathrm{l} 2,4-\mathrm{D}+1 \mathrm{ml} / \mathrm{l} \mathrm{BAP}$ & $\begin{array}{c}\text { Local Yella } \\
\text { Kranti } \\
\text { JD-6 } \\
\text { NDRE-22 }\end{array}$ & $\begin{array}{l}26.89 \\
\\
28.56 \\
27.78 \\
28.78\end{array}$ & $\begin{array}{l}18.89(25.75) \\
17.78(24.92) \\
16.67(24.03) \\
11.11(19.42)\end{array}$ \\
\hline$\overline{T_{2}}$ & $\mathrm{MS}+2 \mathrm{ml} / \mathrm{l} 2,4-\mathrm{D}+1 \mathrm{ml} / \mathrm{l} \mathrm{BAP}$ & $\begin{array}{c}\text { Local Yella } \\
\text { Kranti } \\
\text { JD-6 } \\
\text { NDRE-22 }\end{array}$ & $\begin{array}{l}26.11 \\
\\
28.22 \\
27.56 \\
28.44\end{array}$ & $\begin{array}{l}66.67(56.88) \\
62.22(52.10) \\
65.56(54.10) \\
47.78(43.72)\end{array}$ \\
\hline $\mathbf{T}_{3}$ & $\mathrm{MS}+3 \mathrm{ml} / 12,4-\mathrm{D}+1 \mathrm{ml} / \mathrm{l} \mathrm{BAP}$ & $\begin{array}{c}\text { Local Yella } \\
\text { Kranti } \\
\text { JD-6 } \\
\text { NDRE-22 }\end{array}$ & $\begin{array}{l}23.78 \\
28.22 \\
25.89 \\
27.89\end{array}$ & $\begin{array}{l}72.22(58.26) \\
66.67(54.75) \\
68.89(56.14) \\
57.78(49.49)\end{array}$ \\
\hline $\mathbf{T}_{4}$ & $\mathrm{~B}_{5}+1 \mathrm{ml} / \mathrm{l} 2,4-\mathrm{D}+1 \mathrm{ml} / 1 \mathrm{BAP}$ & $\begin{array}{c}\text { Local Yella } \\
\text { Kranti } \\
\text { JD-6 } \\
\text { NDRE-22 }\end{array}$ & $\begin{array}{l}28.11 \\
\\
28.78 \\
28.22 \\
29.22\end{array}$ & $\begin{array}{l}28.89(32.45) \\
26.67(31.06) \\
31.77(27.78) \\
16.67(24.03)\end{array}$ \\
\hline $\mathbf{T}_{5}$ & $\mathrm{~B}_{5}+2 \mathrm{ml} / 12,4-\mathrm{D}+1 \mathrm{ml} / \mathrm{l} \mathrm{BAP}$ & $\begin{array}{c}\text { Local Yella } \\
\text { Kranti } \\
\text { JD-6 } \\
\text { NDRE-22 }\end{array}$ & $\begin{array}{l}25.44 \\
27.89 \\
27.45 \\
28.78\end{array}$ & $\begin{array}{l}64.24(55.10) \\
55.56(48.22) \\
58.89(51.45) \\
46.67(43.09)\end{array}$ \\
\hline $\mathrm{T}_{6}$ & $\mathrm{~B}_{5}+3 \mathrm{ml} / 12,4-\mathrm{D}+1 \mathrm{ml} / \mathrm{l} \mathrm{BAP}$ & $\begin{array}{c}\text { Local Yella } \\
\text { Kranti } \\
\text { JD-6 } \\
\text { NDRE-22 }\end{array}$ & $\begin{array}{l}23.33 \\
27.11 \\
25.22 \\
27.78\end{array}$ & $\begin{array}{l}71.11(57.59) \\
65.56(54.16) \\
67.78(55.46) \\
47.78(45.64)\end{array}$ \\
\hline & $\begin{array}{c}\text { S.Ed. }( \pm) \\
\text { C.D. }(0.05)\end{array}$ & & $\begin{array}{l}0.61 \\
1.22\end{array}$ & $\begin{array}{l}2.60 \\
\text { NS }\end{array}$ \\
\hline
\end{tabular}


Int.J.Curr.Microbiol.App.Sci (2018) 7(4): 8-19

Table.4 Response of genotypes on shoot initiation

\begin{tabular}{|c|c|c|c|c|c|c|c|}
\hline \multirow[t]{2}{*}{ Genotypes } & \multirow{2}{*}{$\begin{array}{c}\text { Days to } \\
\text { shoot } \\
\text { initiation }\end{array}$} & \multicolumn{3}{|c|}{ Per cent $(\%)$ shoot initiation after } & \multicolumn{3}{|c|}{$\begin{array}{c}\text { Length }(\mathrm{cm}) \text { of regenerated shoot } \\
\text { after }\end{array}$} \\
\hline & & 15 DAI & 30 DAI & 45 DAI & 30 DAI & 45 DAI & 60 DAI \\
\hline $\begin{array}{l}\text { Local } \\
\text { Yella }\end{array}$ & 24.59 & 0.00 & $\begin{array}{c}51.48 \\
(45.86)\end{array}$ & $\begin{array}{c}54.07 \\
(47.38)\end{array}$ & $\begin{array}{c}0.55 \\
(1.02)\end{array}$ & $\begin{array}{l}1.30 \\
(1.34)\end{array}$ & $\begin{array}{c}2.55 \\
(1.83)\end{array}$ \\
\hline Kranti & 27.41 & 0.00 & $\begin{array}{c}48.15 \\
(44.58)\end{array}$ & $\begin{array}{c}54.82 \\
(47.83)\end{array}$ & $\begin{array}{l}0.45 \\
(0.97)\end{array}$ & $\begin{array}{c}1.09 \\
(1.25)\end{array}$ & $\begin{array}{c}2.40 \\
(1.70)\end{array}$ \\
\hline JD-6 & 26.63 & 0.00 & $41.11(39.61)$ & $\begin{array}{c}41.85 \\
(40.04)\end{array}$ & $\begin{array}{c}0.43 \\
(0.96)\end{array}$ & $\begin{array}{c}0.99 \\
(1.22)\end{array}$ & $\begin{array}{c}2.34 \\
(1.69)\end{array}$ \\
\hline NDRE-22 & 28.85 & 0.00 & $\begin{array}{c}30.48 \\
(31.28)\end{array}$ & $\begin{array}{c}33.48 \\
(34.21)\end{array}$ & $\begin{array}{c}0.32 \\
(0.90)\end{array}$ & $\begin{array}{c}0.86 \\
(1.16)\end{array}$ & $\begin{array}{c}2.09 \\
(1.61)\end{array}$ \\
\hline S.Ed. $( \pm)$ & 0.27 & - & 1.92 & 1.43 & 0.02 & 0.02 & 0.06 \\
\hline $\begin{array}{l}\text { C.D. } \\
(0.05)\end{array}$ & 0.55 & - & 3.86 & 2.88 & 0.04 & 0.04 & 0.13 \\
\hline
\end{tabular}

Figures in parentheses are transformed values.

Table.5 Performance of media with different concentrations of phytohormones for shoot initiation

\begin{tabular}{|c|c|c|c|c|c|c|c|c|}
\hline \multirow[t]{2}{*}{ Treatment } & \multirow[t]{2}{*}{$\begin{array}{l}\text { Treatment } \\
\text { composition }\end{array}$} & \multirow{2}{*}{$\begin{array}{c}\text { Days to } \\
\text { shoot } \\
\text { initiation }\end{array}$} & \multicolumn{3}{|c|}{$\begin{array}{c}\text { Per cent (\%) shoot initiation } \\
\text { after }\end{array}$} & \multicolumn{3}{|c|}{$\begin{array}{l}\text { Length }(\mathrm{cm}) \text { of } \\
\text { regenerated shoot after }\end{array}$} \\
\hline & & & $\begin{array}{c}15 \\
\text { DAI }\end{array}$ & 30 DAI & 45 DAI & $\begin{array}{c}30 \\
\text { DAI }\end{array}$ & $\begin{array}{c}45 \\
\text { DAI }\end{array}$ & $\begin{array}{c}\text { 60 } \\
\text { DAI }\end{array}$ \\
\hline $\mathbf{T}_{1}$ & $\begin{array}{c}\mathrm{MS}+4 \mathrm{ml} / \mathrm{l} \mathrm{BAP}+1 \\
\mathrm{ml} / \mathrm{NAA}\end{array}$ & 25.61 & 0.00 & $\begin{array}{c}55.55 \\
(48.25)\end{array}$ & $\begin{array}{c}59.44 \\
(50.54)\end{array}$ & $\begin{array}{c}0.53 \\
(1.01)\end{array}$ & $\begin{array}{c}1.22 \\
(1.29)\end{array}$ & $\begin{array}{c}2.48 \\
(1.73)\end{array}$ \\
\hline$T_{2}$ & $\begin{array}{c}\mathrm{MS}+5 \mathrm{ml} / \mathrm{l} \mathrm{BAP}+1 \\
\mathrm{ml} / 1 \mathrm{NAA}\end{array}$ & 27.25 & 0.00 & $\begin{array}{c}46.86 \\
(43.39)\end{array}$ & $\begin{array}{c}50.56 \\
(45.33)\end{array}$ & $\begin{array}{c}0.39 \\
(0.94)\end{array}$ & $\begin{array}{c}1.01 \\
(1.23)\end{array}$ & $\begin{array}{c}2.29 \\
(1.67)\end{array}$ \\
\hline$\overline{T_{3}}$ & $\begin{array}{c}\mathrm{MS}+6 \mathrm{ml} / \mathrm{l} \mathrm{BAP}+1 \\
\mathrm{ml} / \mathrm{l} \mathrm{NAA}\end{array}$ & 28.70 & 0.00 & $\begin{array}{c}32.78 \\
(33.12)\end{array}$ & $\begin{array}{c}34.10 \\
(35.48)\end{array}$ & $\begin{array}{c}0.27 \\
(0.87)\end{array}$ & $\begin{array}{c}0.80 \\
(1.14)\end{array}$ & $\begin{array}{c}2.13 \\
(1.62)\end{array}$ \\
\hline$\overline{T_{4}}$ & $\begin{array}{c}\mathrm{B}_{5}+4 \mathrm{ml} / \mathrm{l} \mathrm{BAP}+1 \\
\mathrm{ml} / \mathrm{l} \mathrm{NAA}\end{array}$ & 24.47 & 0.00 & $\begin{array}{c}50.00 \\
(45.97)\end{array}$ & $\begin{array}{c}56.11 \\
(48.56)\end{array}$ & $\begin{array}{c}0.66 \\
(1.08)\end{array}$ & $\begin{array}{c}1.33 \\
(1.35)\end{array}$ & $\begin{array}{c}2.58 \\
(1.89)\end{array}$ \\
\hline$T_{5}$ & $\begin{array}{c}\mathrm{B}_{5}+5 \mathrm{ml} / 1 \mathrm{BAP}+1 \\
\mathrm{ml} / \mathrm{l} \mathrm{NAA}\end{array}$ & 26.83 & 0.00 & $\begin{array}{c}44.42 \\
(41.88)\end{array}$ & $\begin{array}{c}36.67 \\
(42.52)\end{array}$ & $\begin{array}{c}0.46 \\
(0.98)\end{array}$ & $\begin{array}{c}1.10 \\
(1.26)\end{array}$ & $\begin{array}{c}2.46 \\
(1.70)\end{array}$ \\
\hline $\mathrm{T}_{6}$ & $\begin{array}{c}\mathrm{B}_{5}+6 \mathrm{ml} / \mathrm{l} \mathrm{BAP}+1 \\
\mathrm{ml} / \mathrm{l} \mathrm{NAA}\end{array}$ & 28.36 & 0.00 & $\begin{array}{c}27.22 \\
(29.40)\end{array}$ & $\begin{array}{c}29.45 \\
(31.76)\end{array}$ & $\begin{array}{c}0.33 \\
(0.91)\end{array}$ & $\begin{array}{c}0.90 \\
(1.18)\end{array}$ & $\begin{array}{c}2.18 \\
(1.64)\end{array}$ \\
\hline & S.Ed. $( \pm)$ & 0.34 & - & 2.35 & 1.75 & 0.02 & 0.03 & 0.08 \\
\hline & .D. $(0.05)$ & 0.68 & - & 4.73 & 3.53 & 0.05 & 0.05 & 0.15 \\
\hline
\end{tabular}

Figures in parenthesis are transformed values. 
Int.J.Curr.Microbiol.App.Sci (2018) 7(4): 8-19

Table.6 Effect of genotype $\times$ treatment on shoot initiation

\begin{tabular}{|c|c|c|c|c|c|c|c|}
\hline \multirow[t]{2}{*}{ Treatment } & \multirow[t]{2}{*}{ Genotypes } & \multirow{2}{*}{$\begin{array}{l}\text { Days to } \\
\text { shoot } \\
\text { initiation }\end{array}$} & \multicolumn{2}{|c|}{$\begin{array}{c}\text { Per cent }(\%) \text { shoot initiation } \\
\text { after }\end{array}$} & \multicolumn{3}{|c|}{ Length of regenerated shoot after } \\
\hline & & & 30 DAI & 45 DAI & 30 DAI & 45 DAI & 60 DAI \\
\hline \multirow[t]{4}{*}{$\mathbf{T}_{1}$} & Local Yella & 23.56 & $60.00(50.81)$ & $66.67(54.81)$ & $0.63(1.06)$ & $1.45(1.40)$ & $2.70(1.79)$ \\
\hline & Kranti & 25.22 & $57.78(49.64)$ & $64.44(53.52)$ & $0.55(1.03)$ & $1.25(1.26)$ & $2.50(1.74)$ \\
\hline & JD-6 & 24.78 & $53.33(46.92)$ & $55.55(48.20)$ & $0.62(1.06)$ & $1.28(1.34)$ & $2.43(1.72)$ \\
\hline & NDRE-22 & 28.89 & $51.11(45.64)$ & $51.11(45.64)$ & $0.30(0.89)$ & $0.90(1.18)$ & $2.30(1.67)$ \\
\hline \multirow[t]{4}{*}{$\mathbf{T}_{2}$} & Local Yella & 24.11 & $51.11(45.64)$ & $53.33(46.92)$ & $0.58(1.04)$ & $1.40(1.38)$ & $2.50(1.74)$ \\
\hline & Kranti & 28.22 & $51.11(45.64)$ & $57.78(49.52)$ & $0.40(0.95)$ & $1.02(1.23)$ & $2.30(1.67)$ \\
\hline & JD-6 & 27.22 & $46.67(43.08)$ & $46.67(43.08)$ & $0.35(0.92)$ & 0.85 (1.16) & 2.28 (1.67) \\
\hline & NDRE-22 & 29.44 & 38.55 (39.19) & $44.45(41.80)$ & $0.22(0.84)$ & $0.78(1.13)$ & $2.08(1.60)$ \\
\hline \multirow[t]{4}{*}{$\mathbf{T}_{3}$} & Local Yella & 27.11 & $46.67(43.08)$ & $46.67(43.08)$ & $0.35(0.92)$ & $0.88(1.18)$ & 2.33 (1.69) \\
\hline & Kranti & 29.22 & $44.44(41.75)$ & $48.89(44.36)$ & $0.25(0.87)$ & $0.85(1.16)$ & $2.27(1.66)$ \\
\hline & JD-6 & 28.78 & $33.33(35.19)$ & $33.33(35.19)$ & $0.28(0.89)$ & 0.78 (1.13) & $2.22(1.65)$ \\
\hline & NDRE-22 & 29.67 & $6.67(12.45)$ & $11.11(19.26)$ & $0.18(0.82)$ & 0.70 (1.09) & $1.72(1.49)$ \\
\hline \multirow[t]{4}{*}{$\mathbf{T}_{4}$} & Local Yella & 22.78 & $57.78(49.53)$ & $57.78(52.09)$ & $0.68(1.09)$ & $1.50(1.41)$ & $2.82(2.33)$ \\
\hline & Kranti & 24.89 & $46.67(46.92)$ & $62.22(52.13)$ & $0.67(1.08)$ & $1.32(1.35)$ & $2.65(1.78)$ \\
\hline & JD-6 & 24.00 & $48.89(44.36)$ & $51.11(45.64)$ & $0.65(1.07)$ & $1.30(1.34)$ & $2.50(1.74)$ \\
\hline & NDRE-22 & 26.22 & $46.67(43.04)$ & $48.89(44.36)$ & $0.63(1.06)$ & $1.20(1.30)$ & $2.37(1.70)$ \\
\hline \multirow[t]{4}{*}{$\mathbf{T}_{5}$} & Local Yella & 23.89 & $48.89(44.36)$ & $51.11(45.64)$ & $0.62(1.06)$ & 1.43 (1.39) & $2.58(1.76)$ \\
\hline & Kranti & 27.78 & $48.89(44.36)$ & $51.11(45.64)$ & $0.48(0.10)$ & $1.22(1.31)$ & $2.37(1.70)$ \\
\hline & JD-6 & 26.89 & $44.44(41.75)$ & $44.44(41.75)$ & $0.38(0.94)$ & 0.88 (1.18) & 2.33 (1.68) \\
\hline & NDRE-22 & 28.78 & $35.44(37.05)$ & $36.44(37.05)$ & $0.37(0.93)$ & 0.88 (1.18) & $2.28(1.67)$ \\
\hline \multirow[t]{4}{*}{$T_{6}$} & Local Yella & 26.11 & $44.44(41.75)$ & $44.44(41.75)$ & $0.45(0.97)$ & $1.15(1.28)$ & $2.40(1.71)$ \\
\hline & Kranti & 29.11 & $40.00(39.19)$ & $44.45(41.80)$ & $0.35(0.92)$ & $0.90(1.18)$ & $2.29(1.68)$ \\
\hline & JD-6 & 28.11 & $20.00(26.36)$ & $20.00(26.36)$ & $0.30(0.89)$ & $0.82(1.15)$ & $2.25(1.66)$ \\
\hline & NDRE-22 & 30.11 & $4.447(10.30)$ & $8.89(17.12)$ & $0.20(0.83)$ & $0.72(1.10)$ & $1.78(1.51)$ \\
\hline & $\begin{array}{l}\text { S.Ed. }( \pm) \\
\text { C.D. }(0.05)\end{array}$ & $\begin{array}{l}0.67 \\
1.35\end{array}$ & $\begin{array}{l}4.70 \\
9.46\end{array}$ & $\begin{array}{l}3.51 \\
7.06\end{array}$ & $\begin{array}{l}0.05 \\
\mathrm{NS}\end{array}$ & $\begin{array}{l}0.05 \\
\mathrm{NS}\end{array}$ & $\begin{array}{l}0.15 \\
\mathrm{NS}\end{array}$ \\
\hline
\end{tabular}

Table.7 Response of genotypes on root initiation

\begin{tabular}{|c|c|c|c|c|c|c|c|}
\hline \multirow[t]{2}{*}{ Genotypes } & \multirow{2}{*}{$\begin{array}{l}\text { Days to } \\
\text { root } \\
\text { initiation }\end{array}$} & \multicolumn{3}{|c|}{ Per cent $(\%)$ root initiation after } & \multicolumn{3}{|c|}{$\begin{array}{c}\text { Length }(\mathrm{cm}) \text { of regenerated root } \\
\text { after }\end{array}$} \\
\hline & & 15 DAI & 30 DAI & 45 DAI & 30 DAI & 45 DAI & 60 DAI \\
\hline $\begin{array}{l}\text { Local } \\
\text { Yella }\end{array}$ & 12.44 & $\begin{array}{c}6.02 \\
(2.16)\end{array}$ & $\begin{array}{l}31.95 \\
(51.11)\end{array}$ & $\begin{array}{c}40.28 \\
(58.88)\end{array}$ & $0.63(1.07)$ & $2.63(1.77)$ & $3.09(1.89)$ \\
\hline Kranti & 12.78 & $\begin{array}{c}6.02 \\
(2.22)\end{array}$ & $\begin{array}{c}34.72 \\
(53.84)\end{array}$ & $\begin{array}{l}43.06 \\
(61.39)\end{array}$ & $0.66(1.07)$ & $2.67(1.78)$ & $3.11(1.90)$ \\
\hline JD-6 & 13.11 & $\begin{array}{c}6.17 \\
(2.35)\end{array}$ & $\begin{array}{c}29.63 \\
(49.14)\end{array}$ & $\begin{array}{c}37.50 \\
(56.43)\end{array}$ & $0.62(1.06)$ & $2.62(1.77)$ & 3.07 (1.89) \\
\hline NDRE-22 & 13.56 & $\begin{array}{c}4.63 \\
(1.97)\end{array}$ & $\begin{array}{c}26.86 \\
(45.74)\end{array}$ & $\begin{array}{l}35.19 \\
(54.26)\end{array}$ & $0.60(1.05)$ & $2.61(1.76)$ & $3.06(1.89)$ \\
\hline $\begin{array}{c}\text { S.Ed. }( \pm) \\
\text { C.D. } \\
(0.05)\end{array}$ & $\begin{array}{l}0.41 \\
0.83\end{array}$ & $\begin{array}{l}0.29 \\
\text { NS }\end{array}$ & $\begin{array}{l}2.12 \\
\text { NS }\end{array}$ & $\begin{array}{l}1.99 \\
\text { NS }\end{array}$ & $\begin{array}{l}0.01 \\
\text { NS }\end{array}$ & $\begin{array}{l}0.01 \\
\text { NS }\end{array}$ & 0.01 \\
\hline
\end{tabular}

Figures in parenthesis are transformed values. 
Table.8 Performance of media with different concentrations of phytohormone for root initiation

\begin{tabular}{|c|c|c|c|c|c|c|c|c|}
\hline \multirow[t]{2}{*}{ Treatment } & \multirow[t]{2}{*}{ Treatment composition } & \multirow[t]{2}{*}{$\begin{array}{l}\text { Days to root } \\
\text { initiation }\end{array}$} & \multicolumn{3}{|c|}{ Per cent $(\%)$ root initiation after } & \multicolumn{3}{|c|}{$\begin{array}{c}\text { Length }(\mathrm{cm}) \text { of regenerated root } \\
\text { after }\end{array}$} \\
\hline & & & 15 DAI & 30 DAI & 45 DAI & 30 DAI & 45 DAI & $60 \mathrm{DAI}$ \\
\hline $\bar{T} T_{1}$ & $\begin{array}{c}\mathrm{MS}+1 \mathrm{ml} / \mathrm{l} \mathrm{NAA}+0.5 \mathrm{ml} / \mathrm{l} \\
\mathrm{BAP}\end{array}$ & 12.97 & $\begin{array}{l}10.42 \\
(3.17)\end{array}$ & $\begin{array}{l}37.50 \\
(56.52)\end{array}$ & $\begin{array}{c}45.83 \\
(63.87)\end{array}$ & $\begin{array}{c}0.78 \\
(1.13)\end{array}$ & $\begin{array}{c}2.82 \\
(1.83)\end{array}$ & $\begin{array}{c}3.42 \\
(1.98)\end{array}$ \\
\hline$\overline{T_{2}}$ & $\begin{array}{c}\mathrm{MS}+2 \mathrm{ml} / \mathrm{l} \mathrm{NAA}+0.5 \mathrm{ml} / \mathrm{l} \\
\mathrm{BAP}\end{array}$ & 14.64 & $\begin{array}{c}7.64 \\
(2.78)\end{array}$ & $\begin{array}{l}29.18 \\
(48.71)\end{array}$ & $\begin{array}{c}40.28 \\
(58.90)\end{array}$ & $\begin{array}{c}0.63 \\
(1.06)\end{array}$ & $\begin{array}{c}2.64 \\
(1.77)\end{array}$ & $\begin{array}{c}3.07 \\
(1.89)\end{array}$ \\
\hline $\mathbf{T}_{3}$ & $\begin{array}{c}\mathrm{MS}+3 \mathrm{ml} / \mathrm{l} \mathrm{NAA}+0.5 \mathrm{ml} / \mathrm{l} \\
\mathrm{BAP}\end{array}$ & 16.25 & $\begin{array}{c}2.31 \\
(1.46)\end{array}$ & $\begin{array}{c}34.03 \\
(47.35)\end{array}$ & $\begin{array}{c}37.50 \\
(56.42)\end{array}$ & $\begin{array}{c}0.54 \\
(1.02)\end{array}$ & $\begin{array}{c}2.53 \\
(1.74)\end{array}$ & $\begin{array}{c}2.93 \\
(1.85)\end{array}$ \\
\hline $\mathbf{T}_{4}$ & $\begin{array}{c}\mathrm{B}_{5}+1 \mathrm{ml} / 1 \mathrm{NAA}+0.5 \mathrm{ml} / \mathrm{l} \\
\mathrm{BAP}\end{array}$ & 13.39 & $\begin{array}{c}9.03 \\
(3.07)\end{array}$ & $\begin{array}{c}31.25 \\
(53.81)\end{array}$ & $\begin{array}{l}43.06 \\
(61.39)\end{array}$ & $\begin{array}{c}0.73 \\
(1.11)\end{array}$ & $\begin{array}{c}2.74 \\
(1.80)\end{array}$ & $\begin{array}{c}3.23 \\
(1.93)\end{array}$ \\
\hline $\mathrm{T}_{5}$ & $\begin{array}{c}\mathrm{B}_{5}+2 \mathrm{ml} / \mathrm{l} \mathrm{NAA}+0.5 \mathrm{ml} / \mathrm{l} \\
\mathrm{BAP}\end{array}$ & 16.06 & $\begin{array}{c}4.86 \\
(1.84)\end{array}$ & $\begin{array}{c}31.25 \\
(50.46)\end{array}$ & $\begin{array}{c}37.50 \\
(56.48)\end{array}$ & $\begin{array}{c}0.45 \\
(1.04)\end{array}$ & $\begin{array}{c}2.59 \\
(1.76)\end{array}$ & $\begin{array}{c}2.97 \\
(1.86)\end{array}$ \\
\hline$T_{6}$ & $\begin{array}{c}\mathrm{B}_{5}+3 \mathrm{ml} / \mathrm{l} \mathrm{NAA}+0.5 \mathrm{ml} / \mathrm{l} \\
\mathrm{BAP}\end{array}$ & 17.61 & $\begin{array}{c}0.00 \\
(0.71)\end{array}$ & $\begin{array}{c}23.61 \\
(42.91)\end{array}$ & $\begin{array}{c}29.86 \\
(49.40)\end{array}$ & $\begin{array}{c}0.67 \\
(1.01)\end{array}$ & $\begin{array}{c}2.47 \\
(1.72)\end{array}$ & $\begin{array}{c}2.88 \\
(1.85)\end{array}$ \\
\hline & S.Ed. $( \pm)$ & 0.51 & 0.36 & 2.60 & 2.43 & 0.01 & 0.01 & 0.01 \\
\hline & C.D. $(0.05)$ & 1.02 & 0.72 & 5.23 & 4.89 & 0.03 & 0.02 & 0.02 \\
\hline
\end{tabular}

Table.9 Effect of genotype $\times$ treatment on root initiation

\begin{tabular}{|c|c|c|c|c|c|c|c|c|}
\hline \multirow[t]{2}{*}{ Treatment } & \multirow[t]{2}{*}{ Genotypes } & \multirow{2}{*}{$\begin{array}{l}\text { Days to root } \\
\text { initiation }\end{array}$} & \multicolumn{3}{|c|}{ Per cent $(\%)$ root initiation after } & \multicolumn{3}{|c|}{ Length of regenerated root after } \\
\hline & & & 15 DAI & 30 DAI & 45 DAI & 30 DAI & 45 DAI & 60 DAI \\
\hline \multirow[t]{7}{*}{$\mathrm{T}_{1}$} & Local Yella & 12.78 & $\begin{array}{c}11.11 \\
(3.36)\end{array}$ & $\begin{array}{c}38.89 \\
(57.76)\end{array}$ & $\begin{array}{c}47.22 \\
(65.10)\end{array}$ & $\begin{array}{c}0.78 \\
(1.13)\end{array}$ & $\begin{array}{c}2.80 \\
(1.82)\end{array}$ & $\begin{array}{c}3.43 \\
(1.98)\end{array}$ \\
\hline & Kranti & 12.44 & 11.11 & 41.67 & 50.00 & 0.80 & $\begin{array}{l}(1.02) \\
2.92\end{array}$ & $\begin{array}{c}(1.90) \\
3.47\end{array}$ \\
\hline & & & $(3.00)$ & $(60.23)$ & $(67.5)$ & $(1.14)$ & $(1.85)$ & (1.99) \\
\hline & JD-6 & 13.11 & 11.11 & 36.11 & $44.44(62$. & 0.77 & 2.78 & 3.41 \\
\hline & & & (3.36) & $(55.36)$ & 63) & (1.13) & $(1.82)$ & (1.98) \\
\hline & NDRE-22 & 13.56 & 8.33 & 33.33 & 41.67 & 0.75 & 2.77 & 3.40 \\
\hline & & & $(2.97)$ & $(52.73)$ & $(60.23)$ & $(1.12)$ & $(1.81)$ & $(1.98)$ \\
\hline \multirow[t]{7}{*}{$\mathbf{T}_{2}$} & Local Yella & 14.44 & $\begin{array}{c}5.55 \\
(2.22)\end{array}$ & $\begin{array}{c}30.56 \\
(49.78)\end{array}$ & $\begin{array}{c}41.67 \\
(60.23)\end{array}$ & $\begin{array}{c}0.63 \\
(1.06)\end{array}$ & $\begin{array}{c}2.65 \\
(1.78)\end{array}$ & $\begin{array}{c}3.08 \\
(1.89)\end{array}$ \\
\hline & Kranti & 14.22 & 8.33 & 33.33 & 44.44 & 0.60 & 2.67 & 3.10 \\
\hline & & & $(2.97)$ & $(52.73)$ & $(62.63)$ & $(1.07)$ & $(1.78)$ & $(1.90)$ \\
\hline & JD-6 & 14.67 & 8.33 & 27.78 & 38.89 & 0.62 & 2.63 & 3.07 \\
\hline & & & (2.97) & (47.63) & $(57.60)$ & (1.06) & (1.77) & (1.89) \\
\hline & NDRE-22 & 15.22 & 8.33 & 25.00 & 36.11 & 0.60 & 2.62 & 3.05 \\
\hline & & & (2.97) & (44.68) & (55.13) & (1.05) & (1.77) & (1.88) \\
\hline \multirow[t]{6}{*}{$T_{3}$} & Local Yella & 16.22 & $\begin{array}{c}2.78 \\
(1.47)\end{array}$ & $\begin{array}{c}30.56 \\
(49.78)\end{array}$ & $\begin{array}{c}38.89 \\
(57.60)\end{array}$ & $\begin{array}{c}0.55 \\
(1.03)\end{array}$ & $\begin{array}{c}2.53 \\
(1.74)\end{array}$ & $\begin{array}{c}2.93 \\
(1.85)\end{array}$ \\
\hline & Kranti & 15.44 & $\begin{array}{c}2.78 \\
(1.47)\end{array}$ & $\begin{array}{c}30.55 \\
(50.26)\end{array}$ & $\begin{array}{c}41.67 \\
(60.23)\end{array}$ & $\begin{array}{c}0.57 \\
(1.03)\end{array}$ & $\begin{array}{c}2.55 \\
(1.75)\end{array}$ & $\begin{array}{c}2.95 \\
(1.86)\end{array}$ \\
\hline & JD-6 & 16.56 & 2.78 & 27.78 & 36.11 & 0.53 & 2.52 & 2.92 \\
\hline & & & (1.47) & (47.63) & (55.13) & $(1.02)$ & $(1.74)$ & (1.85) \\
\hline & NDRE-22 & 16.78 & 2.78 & 27.82 & 33.33 & 0.52 & 2.52 & 2.90 \\
\hline & & & (1.47) & (41.73) & (52.73) & (1.01) & $(1.74)$ & (1.84) \\
\hline \multirow[t]{7}{*}{$\mathbf{T}_{4}$} & Local Yella & 13.22 & 8.33 & 36.11 & 44.45 & 0.73 & 2.75 & 3.23 \\
\hline & & & (2.98) & $(54.40)$ & (62.70) & (1.11) & (1.76) & (1.93) \\
\hline & Kranti & 12.78 & 8.33 & 36.11 & 47.22 & 0.75 & 2.48 & 3.25 \\
\hline & & & (2.98) & (55.36) & (65.03) & (1.12) & (1.73) & (1.94) \\
\hline & JD-6 & 15.34 & 11.11 & 33.33 & 41.67 & 0.72 & 2.73 & 3.22 \\
\hline & & & (3.37) & (52.73) & $(60.23)$ & (1.10) & $(1.80)$ & (1.93) \\
\hline & NDRE-22 & 18.89 & 8.33 & 30.56 & 38.89 & 0.70 & 2.72 & 2.97 \\
\hline \multirow{7}{*}{$\mathbf{T}_{5}$} & Local Yella & 15.22 & $\begin{array}{c}(2.90) \\
8.33\end{array}$ & $\begin{array}{l}30.55 \\
30.55)\end{array}$ & 38.89 & 0.60 & $\begin{array}{l}(1.00) \\
2.75\end{array}$ & $\begin{array}{c}(1.00) \\
2.97\end{array}$ \\
\hline & & & $(2.22)$ & $(50.26)$ & $(57.60)$ & (1.05) & (1.76) & (1.86) \\
\hline & Kranti & 14.78 & 5.55 & 36.11 & 41.67 & 0.62 & 2.48 & 2.98 \\
\hline & & & $(2.22)$ & $(54.65)$ & $(60.23)$ & (1.06) & (1.73) & (1.87) \\
\hline & JD-6 & 15.34 & 5.55 & 30.56 & 36.11 & 0.58 & 2.58 & 2.97 \\
\hline & & & $(2.22)$ & (49.78) & (55.36) & (1.04) & (1.76) & (1.86) \\
\hline & NDRE-22 & 18.89 & $\begin{array}{c}0.00 \\
(0.71)\end{array}$ & $\begin{array}{c}27.78 \\
(47.15)\end{array}$ & $\begin{array}{c}33.33 \\
(52.73)\end{array}$ & $\begin{array}{c}0.57 \\
(1.03)\end{array}$ & $\begin{array}{c}2.57 \\
(1.75)\end{array}$ & $\begin{array}{c}2.95 \\
(1.86)\end{array}$ \\
\hline \multirow[t]{8}{*}{$\overline{T_{6}}$} & Local Yella & 17.00 & 0.00 & 25.00 & 30.56 & 0.60 & 2.47 & 2.88 \\
\hline & & & $(0.71)$ & (44.68) & $(50.10)$ & (1.05) & (1.72) & (1.84) \\
\hline & Kranti & 16.78 & 0.00 & 30.56 & 33.33 & 0.55 & 2.48 & 2.90 \\
\hline & & & $(0.71)$ & (49.78) & (52.73) & (1.03) & (1.73) & (1.84) \\
\hline & JD-6 & 17.44 & 0.00 & 22.22 & 27.78 & 0.48 & 2.45 & 2.87 \\
\hline & & & $(0.71)$ & (41.73) & (47.63) & $(0.99)$ & (1.72) & (1.84) \\
\hline & NDRE-22 & 19.22 & 0.00 & 16.67 & 27.78 & 0.48 & 2.47 & 2.97 \\
\hline & & & $(0.71)$ & (35.44) & (47.15) & $(0.99)$ & $(1.72)$ & (1.86) \\
\hline \multirow{2}{*}{\multicolumn{2}{|c|}{$\begin{array}{l}\text { S.Ed. }( \pm) \\
\text { C.D. }(0.05)\end{array}$}} & 1.01 & 0.72 & & & 0.03 & 0.02 & 0.02 \\
\hline & & NS & NS & 10.46 & 9.79 & NS & NS & NS \\
\hline
\end{tabular}

Figures in parentheses are transformed values. 


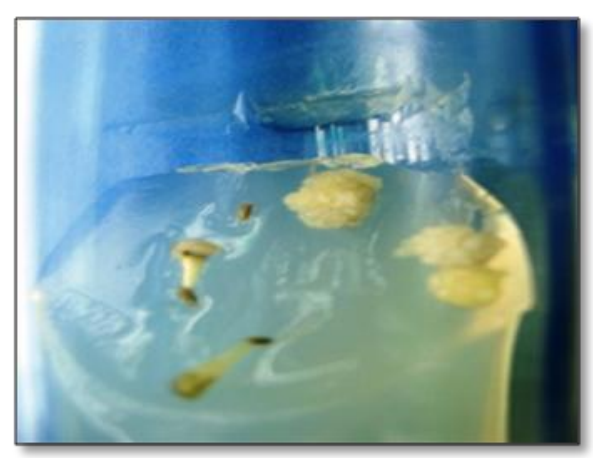

Plate.1 Callus initiation from anther of the genotype (A) Local Yella

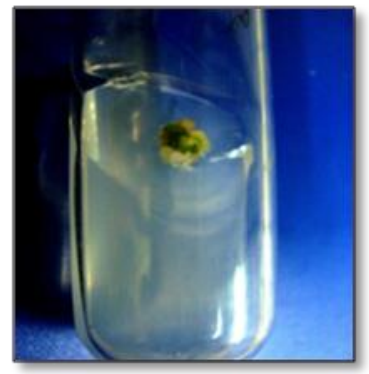

Plate.3 Shoot initiation in Local Yella

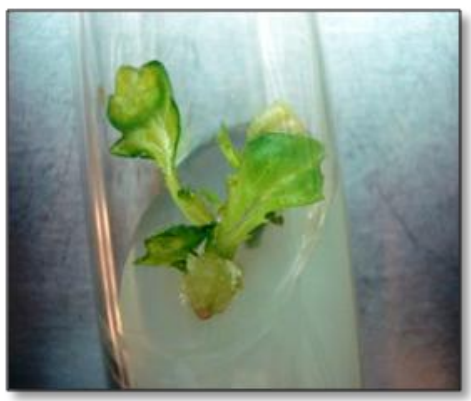

Plate.5 Shoot regeneration in Local Yella

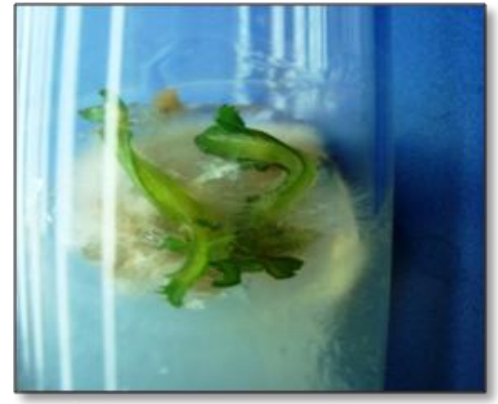

Plate.7 Root regeneration in Local Yella

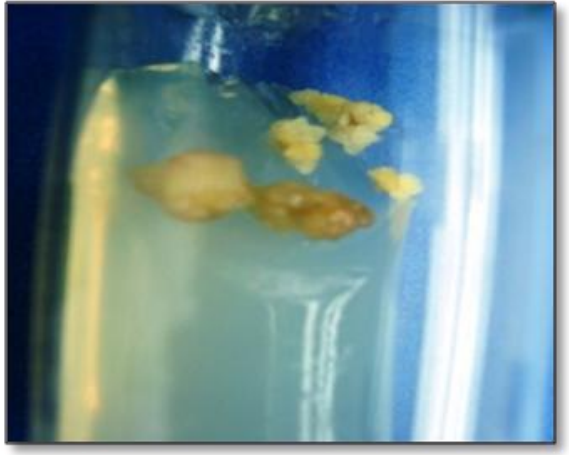

Plate.2 Callus initiation from anther of the genotype (B) Kranti

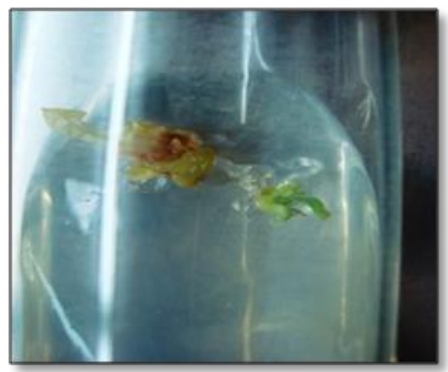

Plate.4 Shoot initiation in Kranti

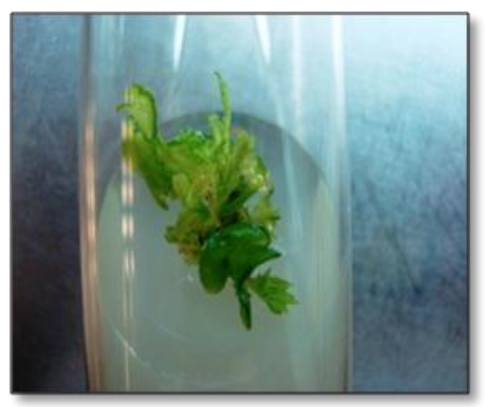

Plate.6 Shoot regeneration in Kranti

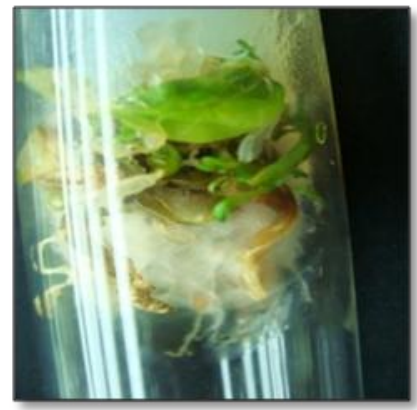

Plate.8 Root regeneration in Kranti 
Plate.9 Chromosome seen under the microscope for the genotypes : (A) JD-6 (B) Kranti (C) Local Yella

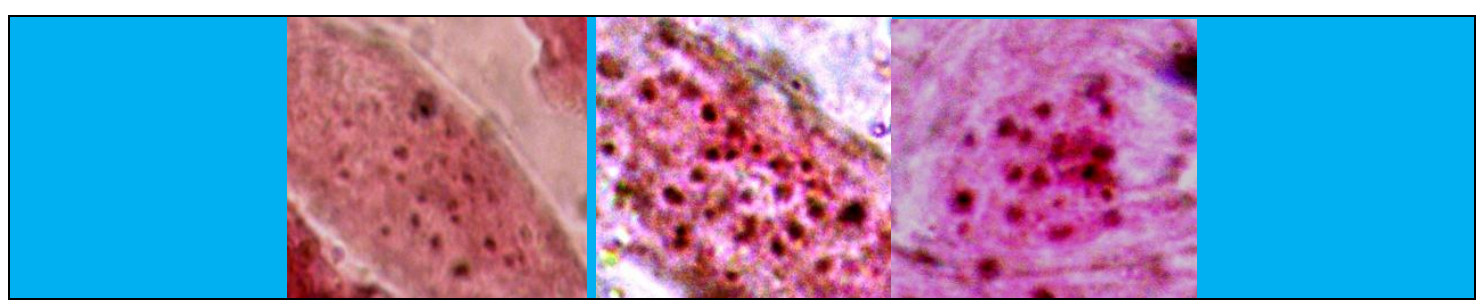

Among the treatments, the media containing $\mathrm{MS}+4 \mathrm{ml} / \mathrm{l} \mathrm{BAP}+1 \mathrm{ml} / \mathrm{l} \mathrm{NAA}$ induced the highest percentage of calli producing shoots (55.55\% and $59.44 \%)$ followed by $\mathrm{B}_{5}+4 \mathrm{ml} / \mathrm{l}$ $\mathrm{BAP}+1 \mathrm{ml} / \mathrm{l} \mathrm{NAA}(50.00 \%$ and $56.11 \%), \mathrm{B}_{5}$ $+5 \mathrm{ml} / 1 \mathrm{BAP}+1 \mathrm{ml} / 1 \mathrm{NAA}(44.42 \%$ and $36.67 \%), \mathrm{MS}+5 \mathrm{ml} / \mathrm{l} \mathrm{BAP}+1 \mathrm{ml} / \mathrm{l} \mathrm{NAA}$ $(46.86 \%$ and $50.56 \%)$ at 30 and 45 DAI respectively as shown in Table 5 . This result is similar with the findings of Wang et al., 2000 and Alam et al., 2009. The media containing BAP at $4 \mathrm{ml} / \mathrm{l}$ induced the highest per cent of shoot initiation and it differed significantly compared to the rest of the treatments. This result is also in consonance with the finding of George et al., (2008); who, revealed that BAP is most effective in promoting differentiation of cell into shoot initials followed by formation of shoot. However, the per cent of shoot initiation decreased in the media containing BAP above $5 \mathrm{mg} / \mathrm{l}$. This is in agreement with Ravanfar et al., 2009 who reported that above $5 \mathrm{mg} / \mathrm{l}$, the mean number of shoots formed per explant decreased and became toxic to the shoot growth. In case of interaction, it was observed that per cent for shoot initiation was found to be significant indicating significant difference among the interaction. At 30 and 45 DAI, all the four genotypes (Local Yella, Kranti, JD-6 and NDRE-22) produced the highest per cent of shoot in MS + $4 \mathrm{ml} / \mathrm{l} \mathrm{BAP}+1 \mathrm{ml} / \mathrm{l} \mathrm{NAA}$ $\{(60.00 \%$ and $66.67 \%), \quad(57.78 \%$ and $64.44 \%),(53.33 \%$ and $55.55 \%)$ and $(51.11 \%$ and $51.11 \%)\}$ followed by B5 $+4 \mathrm{ml} / \mathrm{l} \mathrm{BAP}+$ $1 \mathrm{ml} / \mathrm{l} \mathrm{NAA}\{(57.78 \%$ and $57.78 \%),(46.67 \%$ and $62.22 \%)$, (48.89\% and $51.11 \%)$ and (46.67\% and $48.89 \%)\}$ respectively. Among them, Local Yella $\times$ MS + 4m1/l BAP + $1 \mathrm{ml} / 1$ NAA produced highest percentage of shoots $(60.00 \%$ and $66.67 \%)$ followed by Krantix $\mathrm{MS}+4 \mathrm{ml} / \mathrm{l} \mathrm{BAP}+1 \mathrm{ml} / \mathrm{l} \mathrm{NAA}(57.78 \%$ and $64.44 \%$ ) at both 30 and 45 DAI; whereas, the lowest per cent of shoot was found in NDRE$22 \times \mathrm{B} 5+6 \mathrm{ml} / \mathrm{l} \mathrm{BAP}+1 \mathrm{ml} / \mathrm{l} \mathrm{NAA}(4.45 \%$ and $8.89 \%)$ followed by NDRE- $22 \times \mathrm{MS}+6$ $\mathrm{ml} / \mathrm{l} \mathrm{BAP}+1 \mathrm{ml} / \mathrm{l} \mathrm{NAA}(6.67 \%$ and $11.11 \%)$ at both 30 and 45 DAI respectively (Table 6). This result indicated that interaction between genotype and treatment played a vital role for shoot regeneration which confirms the findings of Khan et al., 2009 and Sayem et al., 2010. Length of regenerated shoot was found to be significant for genotypes and treatments at 30, 45 and 60 DAI. The length of the regenerated shoot was found to be more in Local Yella at 30, 45 and 60 DAI $(0.55 \mathrm{~cm}$, $1.30 \mathrm{~cm}$ and $2.55 \mathrm{~cm}$ respectively). Maximum shoot length at 30,45 and 60 DAI was observed in the media containing B5 + $4 \mathrm{ml} / \mathrm{l}$ $\mathrm{BAP}+1 \mathrm{ml} / 1 \mathrm{NAA}(0.66 \mathrm{~cm}, 1.33 \mathrm{~cm}$ and $2.58 \mathrm{~cm}$ ) followed by $\mathrm{MS}+4 \mathrm{ml} / \mathrm{l} \mathrm{BAP}+1$ $\mathrm{ml} / \mathrm{l}$ NAA $(0.53 \mathrm{~cm}, 1.22 \mathrm{~cm}$ and $2.58 \mathrm{~cm})$, respectively. However, no significant variation was observed for the interaction of genotype and treatment.

\section{Root initiation}

Data on root initiation were found to be significant among genotypes for days to root initiation. Local Yella (12.44 days) took 
minimum time for root initiation while NDRE-22 took maximum time (13.56 days). But the genotypes were found to be nonsignificant for per cent of root initiation at 15 , 30 and 45 DAI and also the length of the regenerated root after 30, 45 and 60 DAI (Table 7). Significant variations were observed amongst the treatments for different parameters of root initiation like days to root initiation, per cent of root initiation at 15,30 and $45 \mathrm{DAI}$ and the length of the regenerated root after 30, 45 and 60 DAI. MS + $1 \mathrm{ml} / 1$ $\mathrm{NAA}+0.5 \mathrm{ml} / \mathrm{l} \mathrm{BAP}$ required minimum time (12.97 days) for root initiation and also produced highest percentage of root initiation in all 15, 30 and 45 DAI $(10.42 \%, 37.50 \%$ and $45.83 \%$ respectively). The length of the regenerated root was also found to be more in this medium $(0.78 \mathrm{~cm}, 2.82 \mathrm{~cm}$ and $3.42 \mathrm{~cm})$ followed by $\mathrm{B}_{5}+1 \mathrm{ml} / 1 \mathrm{NAA}+0.5 \mathrm{ml} / \mathrm{l} \mathrm{BAP}$ $(0.73 \mathrm{~cm}, 2.74 \mathrm{~cm}$ and $3.23 \mathrm{~cm})$ at 30,45 and 60 DAI respectively (Table 8 ).

The interaction was found to have nonsignificant effect for days to root initiation and length of regenerated root at 30,45 and 60 DAI indicating that the genotypes and treatments have independent effects. Whereas the interaction of genotype $\times$ treatment showed significant variation for per cent of root initiation at 30 and 45 DAIAll the genotypes (Local Yella, Kranti, JD-6 and NDRE-22) produced maximum percentage of root in the media containing $\mathrm{MS}+1 \mathrm{ml} / \mathrm{l}$ $\mathrm{NAA}+0.5 \mathrm{ml} / \mathrm{l}$ BAP $\{(38.89 \%, 47.22 \%)$, $(41.67 \%, 50.00 \%),(36.11 \%, 44.44 \%)$ and $(33.33 \%$ and $41.67 \%)\}$ followed by $\mathrm{B}_{5}+1$ $\mathrm{ml} / \mathrm{l} \mathrm{NAA}+0.5 \mathrm{ml} / \mathrm{l}$ BAP $\{(36.11 \%$, $44.45 \%), \quad(36.11 \%, \quad 47.22 \%), \quad(33.33 \%$, $41.67 \%)$ and $(30.56 \%, 38.89 \%)\}$ at 30 and 45 DAI respectively. Among them, Kranti $\times$ MS $+1 \mathrm{ml} / 1 \mathrm{NAA}+0.5 \mathrm{ml} / 1 \mathrm{BAP}$ produced the highest percentage of root $(41.67 \%, 50.00 \%)$ followed by Local Yella $\times$ MS + $1 \mathrm{ml} / 1 \mathrm{NAA}$ $+0.5 \mathrm{ml} / \mathrm{l}$ BAP $(38.89 \%, 47.22 \%)$; whereas, $\mathrm{NDRE}-22 \times \mathrm{B}_{5}+3 \mathrm{ml} / \mathrm{l} \mathrm{NAA}+0.5 \mathrm{ml} / \mathrm{l} \mathrm{BAP}$ produced the lowest percentage of root $(16.67 \%$ and $27.78 \%)$ at 30 and 45 DAI respectively (Table 9).

\section{Cytological analysis}

The root tips of the plants regenerated from the callus of anther culture were found to be haploids, that is, $n=16(2 n=36)$ in nature by cytological examination which confirms the findings of Keller and Armstrong (1983) and, Prabhudesai and Bhaskaran (1993) who revealed that the plants regenerated from the anther culture were haploids in nature.

From the results of this experiment, it can be concluded that among the genotypes, Local Yella was found to be best in all the cases that is, for callus induction, shoot regeneration and root initiation. Among the treatments, the media containing $\mathrm{B}_{5}+3 \mathrm{ml} / 12,4-\mathrm{D}+1 \mathrm{ml} / \mathrm{l}$ $\mathrm{BAP}$ and $\mathrm{MS}+3 \mathrm{ml} / \mathrm{l}$ 2,4-D + $1 \mathrm{ml} / 1 \mathrm{BAP}$ performed best for callus induction. For shoot regeneration, $\mathrm{MS}+4 \mathrm{ml} / \mathrm{l} \mathrm{BAP}+1 \mathrm{ml} / \mathrm{l} \mathrm{NAA}$ followed by $\mathrm{B}_{5}+4 \mathrm{ml} / \mathrm{l} \mathrm{BAP}+1 \mathrm{ml} / \mathrm{l} \mathrm{NAA}$ was found to be best and for root initiation, the media containing $\mathrm{MS}+1 \mathrm{ml} / \mathrm{l} \mathrm{NAA}+0.5$ $\mathrm{ml} / \mathrm{l} \mathrm{BAP}$ and $\mathrm{B}_{5}+1 \mathrm{ml} / \mathrm{l} \mathrm{NAA}+0.5 \mathrm{ml} / \mathrm{l}$ BAP performed best.

\section{References}

Alam, M.A., Haque, M.A., Hossain, M.R., Sarker, S.C. and Afroz, R. (2009). Haploid plantlet regeneration through anther culture in oilseed Brassica species. Bangladesh Journal of Agricultural Research, 34(4): 698-703.

Anonymous, 2013.Rapeseed-mustard production technology for different agro-ecological system in India. Directorate of Rapeseed-Mustard Research, Bharatpur 321 303, India, pp.54.

Chopra, V.L. and Prakash, S (1991). Taxonomy, distribution and 
cytogenetics. In: V.L. Chopra, S. Prakash (eds.) Brassica Oilseed in Indian Agriculture. Vikas Pub. House Pvt. Ltd., New Delhi, pp. 257-304.

Darlington, D.C. and Lacour, L.F. (1976). The Handling of chromosomes. ( $6^{\text {th }}$ edn.) Allen and Unwin, London.

Gamborg, O.L., Miller, R.A. and Ojima, K. (1968). Nutrient requirements of suspension cultures of soybean root cells. Experiment Cell Research, 50: 151-158.

George, E.F., Hall, M.A. and Klerk, G.J.D. (2008). Plant Propagation by Tissue Culture: Volume 1. The Background. Third Edition, Springer Publisher: Dordrecht; London.

Keller, W.A. and Armstrong, K.C. (1983).Production of haploids via anther culture in Brassica oleracea var. italica. Euphytica, 32:151-159.

Khan, M.M.A., Hassan, L., Ahmad, S.D., Shah, A.H. and Batool, F. (2009). In vitro regeneration potentiality of oilseed Brassica genotypes with differential BAP concentration. Pakistan Journal Botany, 41(3): 1233-1239.

Morrison, R.A. and Evans, D.A. (1988). Haploid plants from tissue culture: new plants varieties in a shortened time frame. Biotechnology, 6: 684-690.

Murashige, T. and Skoog, F. (1962).A revised medium for rapid growth and bioassay with tobacco tissue culture. Physiologia Plantarum, 15: 473-497.
Narasimhulu, S.B. and Chopra, V.L. (1987). Tissue culture studies in Brassica carinata A. Braun. Cruciferae Newsletter, 12(1):70.

Ockendon, D.J. and McCleriaghan, R. (1993). Effect of silver nitrate and 2,4-D on anther culture of Brussels sprouts (Brassica olerecea). Plant Cell, Tissue and Organ Culture, 32(1): 41-46.

Prabhudesai, V. and Bhaskaran, S. (1993).A continuous culture system of direct somatic embryogenesis in microsporederived embryos of Brassica juncea. Plant Cell Reports, 12(11): 289-292.

Ravanfar, S.A., Aziz, M.A., Kadir, M.A., Rashid, A.A. and Sirchi, M.H.T. (2009).Plant regeneration of Brassica oleracea subsp. italica (Broccoli) cv. Green Marvel as affected by plant growth regulators. African Journal of Biotechnology, 8(11): 2523-2528.

Sayem, M.A., Maniruzzaman; Siddique, S.S. and Al-Amin, M. (2010).In vitro shoot regeneration through anther culture of Brassica spp. Bangladesh Journal of Agricultural Research, 35(2): 331-341.

Wang, J.X., Sun, Y., Cui, G.M., Liu, S.X., Wang, G.P., Shang, Y.J. and Wang, H. (2000).Effects of plant growth regulators on the differentiation of in vitro-cultured hypocotyls of rapeseed (Brassica).Chinese Journal of oil crop Sciences, 22(1):11-13.

\section{How to cite this article:}

Reetisana, N., Th. Renuka Devi, H. Nanita Devi, J.M. Laishram and Artibashisha Hijam Pyngrope. 2018. In vitro Haploid Plantlet Regeneration through Anther Culture in Locally Adapted Cultivar of Indian Mustard (Brassica juncea L. Czern and Coss). Int.J.Curr.Microbiol.App.Sci. 7(04): 8-19. doi: https://doi.org/10.20546/ijcmas.2018.704.002 